\title{
Index Monitoring System on Aerobic Metabolism Capacity Based on MS.NET Framework
}

\author{
Guoquan Zhang \\ Department of Physical Education, Bohai University, Jinzhou, 121013, China \\ 673722120@qq.com
}

Keywords: MS.NET framework; aerobic metabolism capacity; monitoring system; functional design

\begin{abstract}
MS.Net framework has a cross-language, cross-platform, security, as well as support of open Internet standards and protocols, and other advantages, it is the ideal framework for a new generation of Internet integrated service platform to build, based on the framework for the development of aerobic metabolism capacity index monitoring system for improve service level of sports competition. First, analyze and build aerobic metabolism capacity monitoring indicator system; then, research software system functions and operational data from the "Athlete information management, Sampling information management and Index maintenance management" and the like; and finally, research the MS.NET architecture and describes MS.NET Framework class library and Common language runtime two components. This paper studies the development system, which has the advantages of high code reuse, easy maintenance, easy expansion, and so on.
\end{abstract}

\section{Introduction}

The.NET framework of the Microsoft Corp is the second ActiveX technology, the introduction of the latest framework for building a new generation of Internet integration services platform, and allows the application of various systems to communicate and share data through Internet, used to implement XML, Web Services, SOA (Service-Oriented Architecture) and agility techniques. The ultimate goal of.NET is to allow the user to access the required information, files and programs at any place, at any time, using any device. Users do not need to know where these files, only make a request, and all the complexity of the background are completely shielded. Meanwhile, it is easier for the developers to build Web programs and Web services, to further simplify the development of applications [1-3]. This paper based on the MS.NET framework to develop software systems for monitoring indicators of aerobic metabolism capacity, always find problems in these indicators, good for lowering blood pressure, lowering blood lipids and glucose control, can make a person's psychological changes, so that people cheerful enhance confidence in life, improve immune function and reduce the impact on human carcinogens [6]. Monitoring through indicators, promoting people to participate in aerobic exercise, but also help enhance physical fitness and stamina, to make people energetic, slow aging and prolong life.

\section{Monitoring Index on Aerobic Metabolism Capacity}

Aerobic metabolism exercise is defined as the form of energy supply in aerobic metabolism during exercise. It is characterized by low intensity, rhythmic, uninterrupted, long duration, easy and convenient, easy to adhere to. Perform aerobic metabolism exercise, one can increase the body's absorption and transport, and utilization of oxygen capacity; on the other hand to be able to promote the body's blood circulation, improve microcirculation and internal environment, increase metabolism, improve brain and vital organs physiology features such as heart and lung. Aerobic energy supply comes mainly from carbohydrates and fats, if obesity can consistently perform aerobic metabolism exercise, can effectively deplete the body excess calories and fat, can lose weight and fitness, but also play a lower blood pressure, lipids, blood glucose and improve the role of immunity in the prevention of atherosclerosis, hypertension, coronary heart disease, stroke, diabetes and cancer and other diseases also benefit. There are many sports in the form of aerobic exercise, such as brisk walking, jogging, cycling, jumping rope, dancing fitness dance, skating, 
swimming, playing hair ball, tai chi, stair climbing, rowing, and so on [5,6]. Studies have shown that aerobic exercise is crucial to guarantee a certain amount of exercise and perseverance. The monitoring index of aerobic metabolism capacity is shown in Table 1.

Table 1. Monitoring index of aerobic metabolism capacity

\begin{tabular}{c|c|l|c|c}
\hline No & Code & \multicolumn{1}{|c|}{ Index name } & Abbreviation & Measure unit \\
\hline 1 & 080101 & Maximal oxygen uptake & $\mathrm{VO}_{2}$ max & $\mathrm{L} / \mathrm{min}$ \\
\hline 2 & 080102 & Relative maximal oxygen uptake & $\mathrm{VO}_{2}$ maxRE & $\mathrm{ml} / \mathrm{Kg} \cdot \mathrm{min}$ \\
\hline 3 & 080103 & $\mathrm{VO}_{2}$ max pleteau duration & $\mathrm{VO}_{2}$ maxPD & $\mathrm{s}$ \\
\hline 4 & 080104 & Physical work capacity 170 & $\mathrm{PWC170}$ & $\mathrm{kg} \cdot \mathrm{m} / \mathrm{min}$ \\
\hline 5 & 080105 & Maximal lactate steady state & $\mathrm{MLSS}$ & $\mathrm{mmol} / \mathrm{L}$ \\
\hline 6 & 080106 & Lactate anaerobic threshold & $\mathrm{LAT}$ & $\mathrm{mmol} / \mathrm{L}$ \\
\hline 7 & 080107 & Ventilation anaerobic threshold & $\mathrm{VT}$ & $\mathrm{L} / \mathrm{min}$ \\
\hline 8 & 080108 & Break point & $\mathrm{BrP}$ & $\mathrm{b} / \mathrm{min}$ \\
\hline 9 & 080109 & Respiratory quotient & $\mathrm{RQ}$ & $\%$ \\
\hline
\end{tabular}

Improve aerobic metabolism capacity training methods are often intermittent training, lactate threshold training and sustained endurance training and altitude training.

(1) Intermittent training, exercise intensity required to close to $80 \%-85 \%$ maximal oxygen uptake intensity or near the anaerobic threshold intensity, duration to be appropriately extended. 2 minutes sports and two minutes rest, four minutes sports and four minutes rest intermittent motion, the impact on skeletal muscle aerobic metabolism significantly help improve oxygen utilization capacity;

(2) Lactate threshold training can improve aerobic oxidation for energy capacity load strength training, is currently the most frequently used as a model. Because lactate threshold there are individual differences in training in order to achieve higher requirements, determination of the best individual athlete's anaerobic threshold, and the use of the individual anaerobic threshold intensity training guide;

(3) Endurance training, it is a method in a relatively long period of time, with the more stable the intensity is not too large, not intermittently continuous practice, is an effective training to improve aerobic metabolism for energy capacity methods. Can improve muscle myoglobin content and muscle glycogen reserves, so that the number of mitochondria in skeletal muscle increases, the volume increases, thereby increasing aerobic metabolism capacity.

(4) Altitude training, aimed at improving the Arly's re-synthesis ability of the athletes body tissues under hypoxic conditions. High altitude hypoxia increased the training, to stimulate capacity increased sugar anaerobic glycolysis. Using high altitude training to improve the ability of cells to acquire and use oxygen, so the body's aerobic metabolism capacity has been strengthened.

\section{System Function and Operation Data}

System function from the perspective of the operation, it includes data "SELECT, UPDATE, INSERT and DELETE" and so on; from the perspective of the user, it includes a "Athlete information management, Sampling information management, Index maintenance management and System management", and so on. Systems management is a regular feature of the system software. This paper only studies the first three functions. That is mainly to complete the "SELECT, UPDATE, INSERT and DELETE" operations of the "Athlete information, Sampling information and Index information". System functions and operating data is shown in Fig. 1.

In Fig. 1, "Athlete information" includes Athlete no, Athlete name, Athlete ID number, Athlete sex, Athlete birthday, Athlete Sports Item, Athlete grade, Athlete phone, Athlete E-mail, and so on; "Sampling information" includes Sampling no, Sampling date, Sampling time, Sampling sites, 
Sampling personnel, Sampling temperature, Sampling humidity, Sampling wind force, Sampling environmental and so on; "Index information" includes Maximal oxygen uptake, Relative maximal oxygen uptake, VO2max plateau duration, Physical work capacity 170, Maximal lactate steady state, Lactate anaerobic threshold, Ventilation anaerobic threshold, Break point, Respiratory quotient and so on.

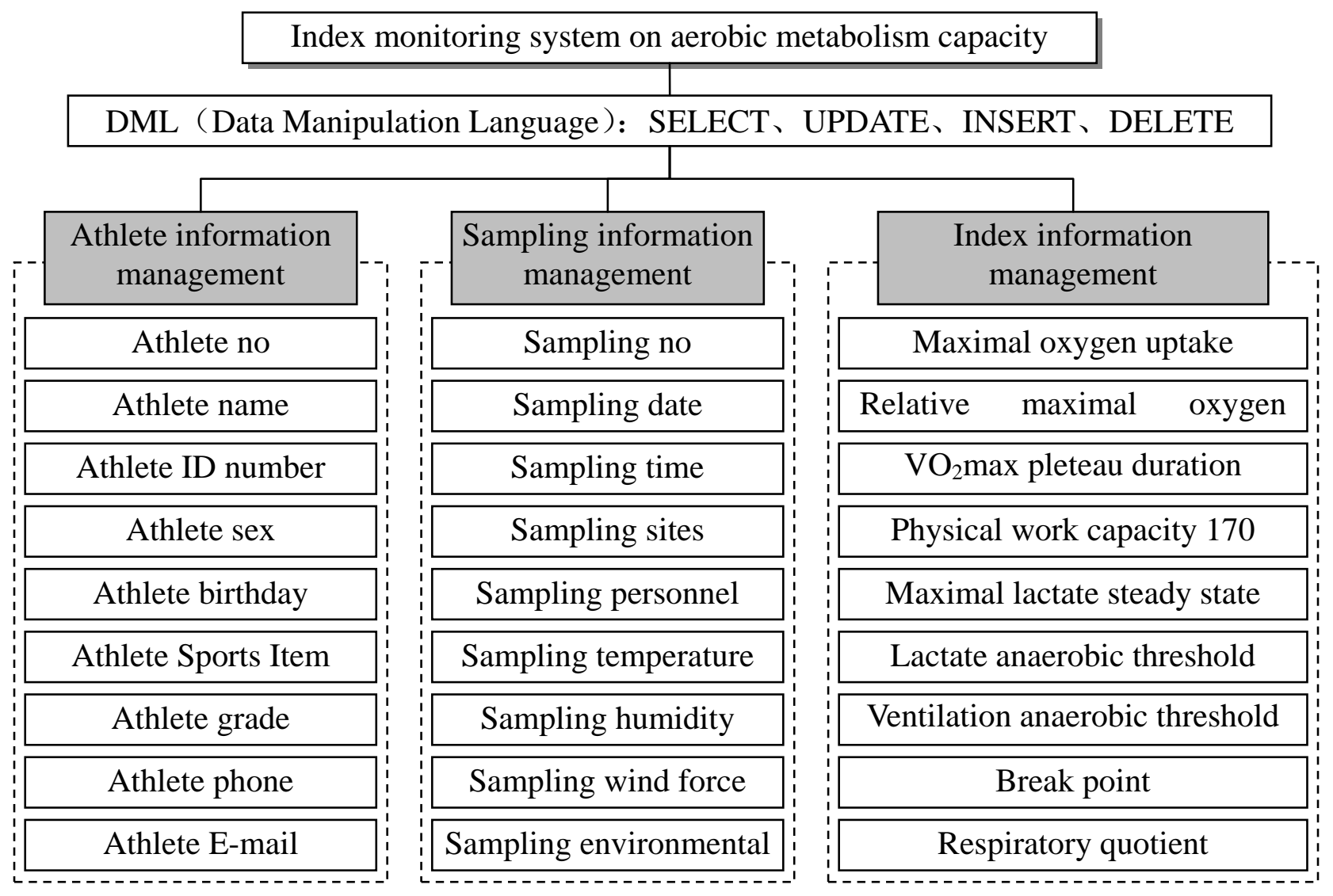

Fig. 1. System function and operational data

\section{Architecture on MS.NET Framework}

MS.NET provides a rich programming model and type library for handling configuration data for the application developer. Have these components, developers and users can easily without the need to recompile the application of the case, through the configuration of the data set, the implementation of the application behavior and the results to intervene, so that the same application in the case of cannot change the source program to meet the specific needs of different application scenarios. For the purposes of the application framework, in most cases, developers need to be able to configure it to obtain a different framework features. MS.NET frame architecture is shown in Fig. 2.

.NET Framework mainly has two components: Framework class library and Common language runtime.NET (CLR) [7-9].

.NET Framework class library is a library, which is composed of a class, interface, and a value type. Can access system functions through the contents of the library, is the basis for generating .NET Framework applications, components and controls. .NET Framework class library include Web Forms, Web Services, Win Forms, ADO.NET and XML Class, Basic framework class. Can develop a graphical user interface (GUI) applications, can also be developed based on ASP.NET Web applications.

Common Language Runtime (CLR) is a run environment, responsible for resource management (memory allocation and garbage collection), and to ensure the necessary separation between the application and the underlying operating system, include Common language specification (CLS) 
and Common Type System (CTS) [8]. The difference of programming language lies not only in the type, some languages support multiple inheritance, some languages support unsigned data types, some of the language supports operator overloading, CLS limits by these various difference caused interoperability problems. CTS not only to achieve a COM-compatible type of variable, but also defined by user-defined type of approach to the type of extension. Any .NET platform as targeting language must establish a mapping between the data type and the CTS type.

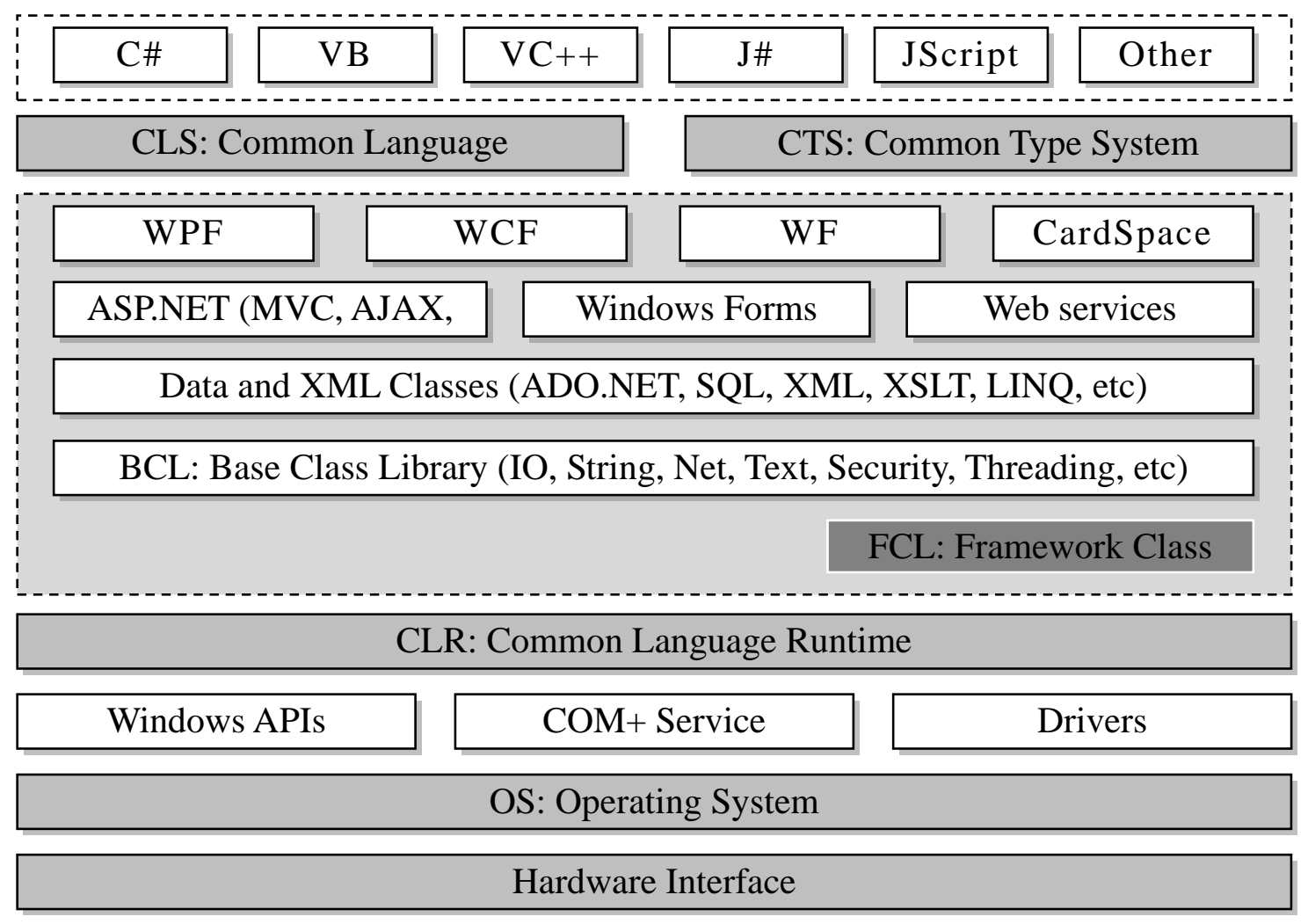

Fig. 2. .NET Framework

\section{Data Access Technology}

Most applications require some form of data access. If you want to create a new application, there are three excellent data access methods to choose from: ADO.NET, ADO and OLE DB. If you need to modify existing data access applications, in order to facilitate maintenance, the current data can continue to use the application access technology. However, if you want the application to have a longer life cycle, you should consider re-designed to the hosted applications using ADO.NET or native applications using ADO. In the long run, newer data access technology is often able to reduce development time, simplify the code and provide good performance [10]. MS.NET recommended accessing the database through ADO.NET.

ADO.NET is an important application-level interface for providing data access services in Microsoft .NET platform. In ADO.NET, you can use the new .NET Framework data providers to access data sources. These data providers include: SQL Server .NET Framework data provider, OLE DB .NET Framework data providers, ODBC .NET Framework data provider, and Oracle .NET Framework data provider.

ADO.NET object model has five main objects, respectively are the Connection objects, Command objects, DataAdapter objects, DataReader objects and DataSet objects. Connection object is used to establish a connection with the data source to access the data source when dealing with the security settings required; Command object to execute SQL commands on data source and returns the results, the results can be filled in the DataReader; DataReader object from the data source to only forward reading to the read-only data stream, the speed of DataReader is faster than DataSet; DataAdapter object provides a Fill method to populate the data source into the DataSet, 
and provides Update method for the modified data in the DataSet update to the data source; DataSet objects support database access no connection, you can access data from multiple heterogeneous data sources. DataSet can be seen as a memory database, a DataSet object can contain several DataTable objects, the actual data stored in the DataTable object. DataSet supports random access.

\section{Conclusion}

MS.Net framework has the advantages of cross-language, cross-platform, security, as well as open Internet standards and protocol support and so on. Specific performance: interoperability support multiple languages, components in a language that is developed, can be in another component through object-oriented inheritance to reuse; be compiled by each language into an intermediate language, and then perform, when using time compiler to compile the local platform code, in order to achieve the interoperability of heterogeneous platform object; run through the common language runtime to achieve the resource objects and type-safe; through HTTP, XML, SOAP, WSDL and other Internet standards support, providing in a heterogeneous network environment to access remote service. But there are only run on the Windows platform, the efficiency is lower than localized compiler, the opening is lower than Java, and other shortcomings. In this paper, based on the MS.NET framework, the development of index monitoring system on aerobic metabolic capacity has been fully utilized, and it has important significance for the objective analysis and evaluation of the physical exercise capacity, to detect the exercise training effect, and the in-depth study of the rules and characteristics of anaerobic metabolism capacity.

\section{References}

[1] H. L. Chen, N. Cui, S. L. Cui, et al., "Design and Realization of University Competition Management System Based on .NET Platform," Computer Technology and Development, vol. 23, no. 1, pp. 173-176, 2013.

[2] T. F. Li, Y. J. Lu, S. B. Du, "Design and Implementation of Data Structureteaching Website Based on .NET," Computer Programming Skills \& Maintenance, vol. 21, no. 6, pp. 121-123, 2014.

[3] Y. X. Zhao, Y. B. Wu, "Design of fast development framework based on.NET," Computer Knowledge and Technology, vol. 10, no. 13, pp. 2996-3000, 2014.

[4] Baidu experience, "The seven benefits on aerobic metabolism sports," http://jingyan.baidu.com/article/92255446ed784b851648f4d5.html, 2015-1-5.

[5] Cao Zhanliang's Blog, "Training of aerobic metabolism capacity," http://blog.sina.com.cn/s/blog_5675ba030101hbol.html, 2015-1-5.

[6] Baidu Encyclopedia, "Aerobic metabolism," http://baike.baidu.com/link?url=vHNda5y4-Id8kAvqoTpfararZdLWBlcM1yhYlb9GmlHgkT1J 007KtrT9Nk3Ig8weQjF-sKlr1W2L_h3gESCJXa, 2015-1-5.

[7] C. Q. Wu, "The Research on Development Mode of Software in .Net Framework," Natural Science Journal of Harbin Normal University, vol. 30, no. 3, pp. 99-102, 2014.

[8] H. W. Zhou, "Development of Web Application Based on ASP. NET MVC Framework," Computer and Modernization, vol. 29, no. 10, pp. 197-199, 2013.

[9] S. Lv, W. S. Chen, "Design and Realization of enterprise management information system based on.Net," Information \& Communications, vol. 28, no. 10, pp. 122-123, 2014.

[10] Microsoft Developer Network, "Data access technology," https://msdn.microsoft.com/zh-cn/library/aa290751, 2015-7-5. 